\title{
Relativistic jets and current driven instabilities
}

\author{
A. Ferrari, A. Mignone and M. Campigotto \\ Dipartimento di Fisica Generale, Università di Torino, \\ Via Pietro Giuria 1, 10125, Torino, Italy \\ email: ferrari@ph.unito.it
}

\begin{abstract}
We review the present results on the study of the propagation of relativistic collimated outflows characteristics of active galaxies and active stars. Magnetic fields, namely their azimuthal components, gives rise to current driven instabilities whose nonlinear development can actually be connected to the complex morphologies observed in astrophysical jets.
\end{abstract}

\section{Introduction}

The first direct evidence of relativistic effects in astrophysics was the discovery of extended radio sources with supersonic jets showing also apparent superluminal motions Cohen et al. (1971). Active galaxies in general, in particular FR II radio galaxies, quasars and blazars, are characterized by the dynamics of powerful outflows with speed close to the velocity of light; in addition the matter of these outflows is relativistic by itself with large Lorentz factors in order to produce non thermal radiation by synchrotron and inverse Compton processes from the radio to the gamma-ray band. The same physics, although on a much smaller scale, underlies the morphologies and radiation characteristics of galactic relativistic stars and X-ray binaries. Recently relativistic jets have been proposed as the basic ingredient to interpret gamma-ray bursts.

The study of the physics of relativistic jets follows three major lines of investigation: (i) acceleration and collimation, (ii) propagation and stability in the interaction with the intergalactic plasma, (iii) termination and momentum/energy release into the external ambient. The current understanding of line (i) is through the so-called magneto-centrifugal mechanism in which a magnetized differentially rotating accretion disk generates Lorentz forces that accelerate a charged particle beam to relativistic velocity along the rotation axis of the system and at the same time keep the beam collimated by the azimuthal magnetic field component. This azimuthal component is convected by the beam while propagating to large scales and is crucial in defining its stability. Several papers have analyzed the relativistic jet stability (for a review see Massaglia et al., 2008). Beam instabilities can be divided in two classes: instabilities due to interaction of the relativistic beam with the external medium and intrinsic instabilities related to the magneto-hydrodynamic structure of the beam. External instabilities are pressure-driven and produce mixing with the ambient with transfer of linear momentum and slowing-down of the flow. Intrinsic instabilities are typically current-driven more prone to affect the morphology of the beam and its radiation emission characteristics.

In this paper we shall concentrate on the case of intrinsic current-driven instabilities, referring to previous papers for external instabilities that are likely to be more important in the termination phases of the jet, (Rossi et al., 2008). Current driven instabilities have been studied in the non-relativistic linear limit by Appl \& Camenzind (1993), while 
relativistic linear studies have been presented by Istomin \& Pariev (1994), (1996). Linear studies are strongly affected by the assumed boundary conditions and cannot follow the complex nonlinear effects that may damp unstable modes. In the following we discuss a series of fully three-dimensional numerical simulations of the propagation of relativistic plasma jets with both axial and azimuthal magnetic field components. Interesting effects of relevance for astrophysical applications are obtained and analyzed.

\section{Numerical Approach}

We study the dynamical evolution of current-carrying jets by solving the three-dimensional time-dependent special relativistic MHD equations:

$$
\begin{array}{ll}
\frac{\partial}{\partial t}(\rho \gamma)+\nabla \cdot(\rho \gamma \boldsymbol{v}) & =0, \\
\frac{\partial \boldsymbol{m}}{\partial t}+\nabla \cdot\left[w \gamma^{2} \boldsymbol{v} \boldsymbol{v}-\boldsymbol{B B}-\boldsymbol{E} \boldsymbol{E}+\mathrm{I}\left(p+p_{E}\right)\right] & =0, \\
\frac{\partial \boldsymbol{B}}{\partial t}+\nabla \times \boldsymbol{E} & =0, \\
\frac{\partial}{\partial t}\left(\gamma^{2} w-p+\frac{p_{E}}{2}\right)+\nabla \cdot \boldsymbol{m} & =0,
\end{array}
$$

where $\rho$ is the rest-mass density, $\gamma$ the Lorentz factor of the flow, $\boldsymbol{m}=w \gamma^{2} \boldsymbol{v}+\boldsymbol{E} \times \boldsymbol{B}$ is the momentum density, $\boldsymbol{B}$ is the magnetic field, $\boldsymbol{E}=-\boldsymbol{v} \times \boldsymbol{B}$ is the electric field (in the infinite conductivity limit) and $w$ is the gas enthalpy. The total pressure $p+p_{E}$ accounts for thermal and electromagnetic contributions with $p_{E}=\boldsymbol{B}^{2} / 2+\boldsymbol{E}^{2} / 2$. The gas enthalpy $w$ is related to $\rho$ and $p$ via the TM equation of state (Mignone \& McKinney, 2007).

We analyze two different configurations consisting, namely, of a full jet (§2.1) and an infinitely long periodic jet $(\S 2.2)$. The system of conservation laws $(2.1)$ is solved using the high-resolution, Godunov-type, PLUTO code (Mignone et al., 2007) for astrophysical gasdynamics.

\subsection{Full Jet Simulations}

In the global simulations, we inject a relativistic beam at the lower $y$ - boundary into a stationary medium with uniform density and pressure. The inflow values are prescribed in terms of the jet-to-ambient density contrast $\eta$, Lorentz factor $\gamma$, sonic and Alfvénic Mach numbers. We consider two different magnetic field topologies. In the first one, the magnetic field is purely toroidal and it is carried along with the beam. Radial profiles are chosen to guarantee force balance between Lorentz force and pressure gradient inside the jet by solving the radial momentum equilibrium equation (2.2), see also Mignone et al. (2010). In the second case, the field is constant and purely poloidal in the direction of jet propagation and it threads the ambient medium as well.

The computational domain is defined by $x, z \in[-L / 2, L / 2], y \in\left[0, L_{y}\right]$ where $L=56$ and $L_{y}=80$. A uniform grid resolution is employed in $y$ and for $|x|,|z|<10$ while geometrically stretched cells are used otherwise. The overall resolution is $640 \times 1600 \times$ 640 , corresponding to the largest resolution numerical simulation of three dimensional relativistic jet carried so far.

\subsection{Infinite Periodic Jet}

The initial condition consists of an equilibrium configuration describing an infinitely long plasma column moving at relativistic speed in the vertical $z$ direction. For simplicity, we 
study the equilibrium by imposing cylindrical symmetry around the axis of propagation (i.e., $\partial / \partial \phi=\partial / \partial z=0$ ) and by setting the radial components of velocity and magnetic field to zero, $v_{r}=B_{r}=0$. With these assumptions the Lorentz and electric forces have only one non-vanishing component along the radial direction and a stationary configuration requires $\nabla \times \boldsymbol{E}=0$ as well as the solution of the $r$-component of the momentum equation:

$$
\frac{\partial p}{\partial r}-\frac{w \gamma^{2} v_{\phi}^{2}}{r}=[\boldsymbol{E} \nabla \cdot \boldsymbol{E}+(\nabla \times \boldsymbol{B}) \times \boldsymbol{B}] \cdot \hat{\mathbf{e}}_{r},
$$

where $\hat{\mathbf{e}}_{r}$ is the unit vector in the radial direction. The previous equation states a mutual balance between pressure, centrifugal, electrical and Lorentz forces. In the following we will assume that pressure forces are negligible $(p=$ cost) and that the longitudinal component of magnetic field is constant, $\partial_{r} B_{z}=0$. Furthermore, we prescribe the radial profiles of $v_{z}, v_{\phi}, B_{z}$ so that direct integration of Eq. (2.2) leads to the following quadratic equation in $B_{\phi}$ :

$$
B_{\phi}^{2}=\left(v_{\phi}-K B_{\phi}\right)^{2} B_{z}^{2}+Q, \quad \text { with } \quad Q=\frac{1}{r^{2}} \int_{0}^{r} 2 w \gamma^{2} v_{\phi}^{2} r d r,
$$

where $K=v_{z} / B_{z}$. The explicit form of $\boldsymbol{v}(r)$ and $B_{z}$ must be properly chosen in order to match the parameter definition,

$$
q=\left.\frac{B_{\phi}}{B_{z}}\right|_{r=\frac{1}{2}}, \quad \gamma=\left.\frac{1}{\sqrt{1-v_{z}^{2}-v_{\phi}^{2}}}\right|_{r=0}, \quad M_{s}^{2}=\frac{w \gamma^{2} \boldsymbol{v}^{2}}{p}, \quad M_{A}^{2}=\frac{w \gamma^{2} \boldsymbol{v}^{2}}{\left\langle\boldsymbol{B}^{2}\right\rangle}
$$

that is, the topology of magnetic field $q$, the Lorentz gamma factor, the kinetic to thermal energy ratio and the kinetic to (average) magnetic energy ratio. We choose the profile of the azimuthal velocity to be

$$
v_{\phi}(r)=v_{\phi, M} \frac{r}{b}\left[1-e^{(r-1)^{3} / a^{3}}\right], \quad v_{z}(r)=\frac{v_{z}(0)}{\cosh \left(r^{4}\right)},
$$

where $a=0.4$ an $b=0.43$ are chosen in such a way that the azimuthal velocity has a maximum in $r \approx \frac{1}{2}$ where $v_{\phi}=v_{\phi, M}$. If $B_{z}$ is known, the explicit value of $v_{\phi, M}$ could be found by solving Eq. (2.3) in $r=\frac{1}{2}$. In practice, however, $B_{z}$ is not known a priori but depends on the velocity through the definition of $M_{A}$ (Eq 2.4) and one has to iterate on the values of $B_{z}$ until the prescribed kinetic to magnetic energy ratio is satisfied.

The computational domain consists of the three-dimensional box $x, y \in[-10,10]$, $z \in[0,10]$ in units of the column radius. The grid resolution is uniform in $z$ and for $|x|,|y|<5$, while it becomes geometrically stretched outside of this region. Employing 20 zones on the column radius and 44 zones to reach the outer boundaries in each directions, we end up with an overall resolution of $288 \times 288 \times 200$ computational cells. Periodic boundary conditions are used in the vertical direction while open boundaries hold at the remaining sides.

\section{Results}

\subsection{Full Jet Simulations}

As shown in (Mignone et al., 2010), the propagation of three-dimensional jet carrying a strong toroidal field bears little resemblance with the corresponding 2-D axisymmetric simulations showing prominent nose cone structures, (Komissarov, 1999; Leismann et al., 2005). Here, instead, the fully developed three dimensional structures becomes unstable 


\begin{tabular}{l|cccccccc} 
& A1 & B1 & B2 & B3 & B4 & B5 & C1 & C2 \\
\hline$\gamma$ & 2 & 5 & 5 & 5 & 5 & 5 & 10 & 10 \\
$M_{A}$ & 1 & 3 & 3 & 3 & 1 & 1 & 3 & 3 \\
$q$ & 4 & 1 & 4 & 10 & 1 & 4 & 4 & 10
\end{tabular}

Table 1. Simulation cases for the periodic jet runs. Here $\gamma, M_{A}$ and $q$ have the meaning given in Eq. (2.4). In all cases, the sonic Mach numbers $M_{s}$ and the density contrast are held fixed to 30 and 1 , respectively.

in proximity of the jet head and the trajectory progressively bends away from the main longitudinal axis. Overally, the backflowing material forms a very asymmetric cocoon as a result of the changes in the direction of the jet head. This is clear by inspecting

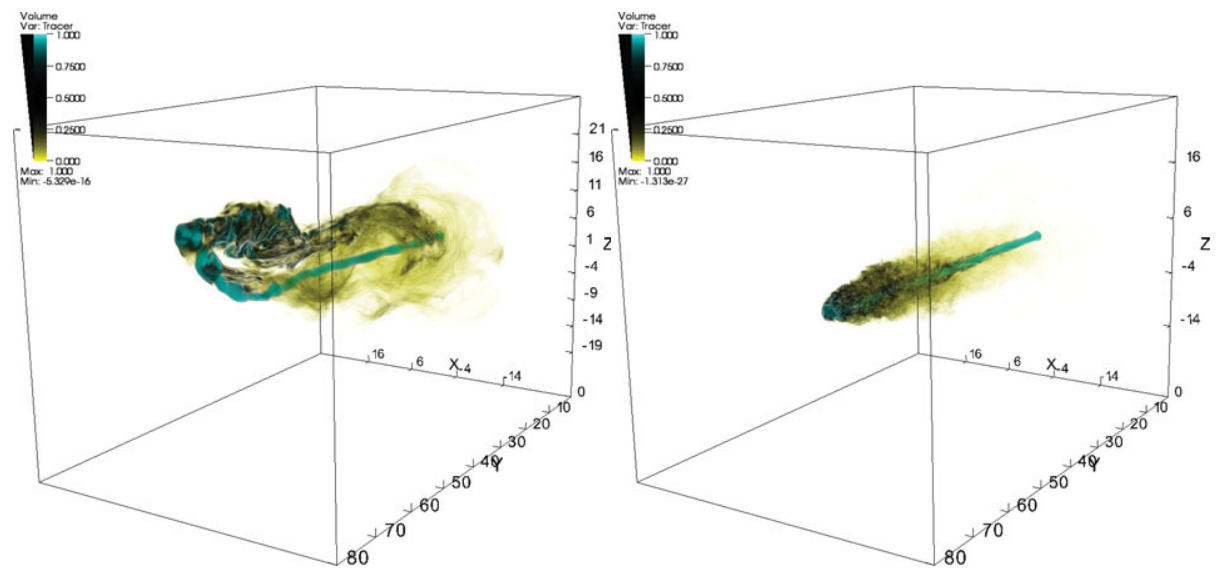

Figure 1. Three-dimensional rendering of the passive scalar in the full jet simulation at $t=770$ for the toroidal jet simulation (left panel) and poloidal (constant vertical field) simulation (right panel).

the left panel in Fig 1 showing the spatial distribution of the passive scalar at $t=770$ (in simulation units). The wandering of the jet head, induced by kink-instability effects, may create multiple sites where the jet impacts on the external medium forming strong shocks. This behaviour may originate the multiple hotspots that are observed in several radiogalaxies.

The toroidal field seems to protect the jet core from any interaction with the surrounding, thus preventing momentum transfer to the external medium. This is due to the presence of low-order modes $(m<2)$ only, producing oval deformations but not inducing any mixing. The tension of the toroidal field, in fact, acts as a strong stabilizing factor for high-order modes and allows to maintain a highly relativistic spine all along its length, (Mignone et al., 2010).

In the case of a purely constant longitudinal field, on the other hand, the results are more similar to the hydro cases discussed by Rossi et al., (2008). Indeed, the presence of a magnetic field does not seem to introduce significant differences with respect to the unmagnetized case and the jet propagates along the main direction axis without suffering from any deflection, see the right panel in Fig 1.

\subsection{Periodic Jet Simulations}

We have carried out numerical simulations using the cases illustrated in Table 3.2. As a general trend, our results indicate that the presence of a relatively larger toroidal 
magnetic field component $(q \gtrsim 1)$ leads to the onset of instability which, in most cases, is triggered by the $m=1$ (kink) unstable mode.

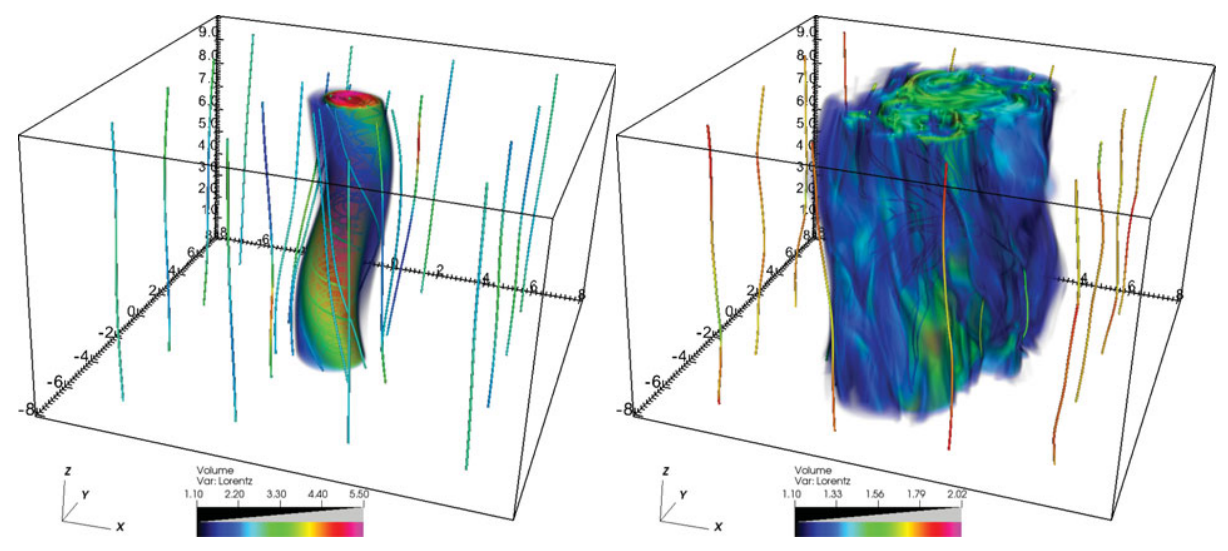

Figure 2. Three-dimensional rendering of the Lorentz factor in the periodic jet simulation for case B2 at $t=75$ (left panel) and $t=225$ (right panel). Magnetic field lines are overplotted.

This behavior can be observed in the left panel of Fig 2 showing prominent wiggling and column deformation at $t=75$ for case B2. The nonlinear development (right panel at $t=200$ ) shows that the jet structure is re-organized on a larger layer with considerable reduction of the Lorentz factor $(\gamma \sim 2$ at the end of simulation) and a more chaotic distribution. A similar behavior is also observed for larger values of $q$ (case B3) and at larger Lorentz factors (cases $\mathrm{C} 1$ and $\mathrm{C} 2$ ). On the other hand, when toroidal and poloidal components are comparable $\left(B_{z} \approx B_{\phi}\right.$ at $r=\frac{1}{2}$ in cases B1 and B4) the column does not show any evidence for instability. See, for instance, the left panel in Fig 3. For larger magnetic field strengths (case B5), we observe a stable behavior even
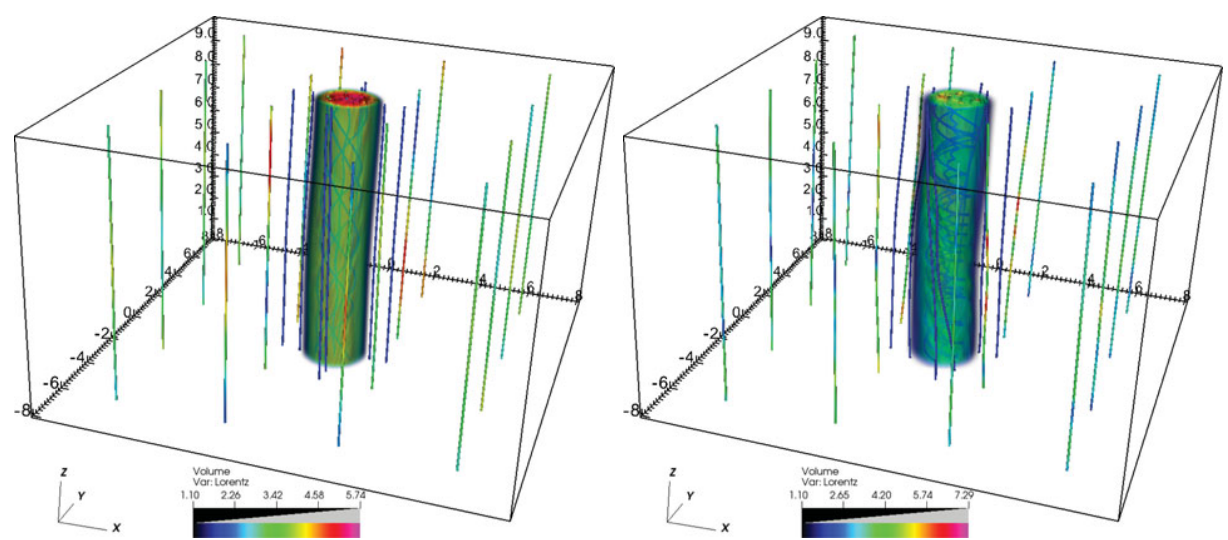

Figure 3. Same as Fig 2 showing the stability of the column for case B1 (left panel, $t=200$ ) and B5 (right panel, $t=320$ ).

in long term integration, as shown in the right panel of Fig 3. In the regime of strong magnetizations, in fact, the system can be well described by the force-free approximation. In this limit, our simulation results are in agreement with the findings of Istomin \& Pariev (1994), (1996) who have shown that a jet with a longitudinal electric current remains stable with respect to helical as well as axially symmetric (pinch) modes. We believe that the induced stability owes to the stabilizing action of the electric field and, therefore, it 
has to be considered as a purely relativistic effect. To support our conclusion, we notice that corresponding weakly relativistic cases (e.g. case A1 with $\gamma=2$ ) are still unstable, as expected on a classical basis.

\section{Summary}

Kink modes in current-driven instabilities appear to be very important in the morphological evolution of relativistic jets. In the full-jet simulations the irregular oscillations in the propagation direction and the spreading of the jet's head are reminiscent of the jets in extended radio galaxies. Large Poynting fluxes associated with azimuthal magnetic field components produce strong kinks and complex head wigglings. On the other hand, large kinetic fluxes avoid kinks and the jet's head proceeds uprightly. The spine of the jet is always highly relativistic on the average, while shocks create intermittent structures. In a separate set of simulations (Rossi et al., 2010) we have verified that when the jet encounters a low density region outside the associated galaxy its structure becomes again straight and organized and kinks disappear.

In order to explore the instability mechanism as a function of the physical parameters (in particular of the Kinetic to Poynting flux ratio $M_{A}$ ), we have performed simulations of an infinite periodic jet. The results indicate that the instability is strong in the nonrelativistic or relativistic cases for $M_{A} \gtrsim 1$, provided the magnetic azimuthal component is stronger than the longitudinal $(q>1)$. Instability becomes weaker for larger field strengths $\left(M_{A} \sim 1\right)$ and tends to disappear when the magnetic field is strong enough $\left(M_{A}\right.$ small) to move the system towards the force-free field limit (Istomin \& Pariev, 1994).

\section{Acknowledgement}

The three-dimensional numerical simulations of the infinite periodic jet have been carried out using the matrix cluster available at the Caspur computing facility.

\section{References}

Appl, S. \& Camenzind, M. 1992, A\& $A$ A, 256, 354

Cohen, M., Canno, W., Purcell, G., Shaffer, D., Broderick, J., Kellermann, K., \& Jauncey, D. 1971, ApJ, 170, 207

Istomin, Y. N. \& Pariev, V. I. 1994, MNRAS, 267, 629

Istomin, Y. N. \& Pariev, V. I. 1996, MNRAS, 281, 1

Komissarov S., 1999, MNRAS, 308, 1069

Leismann T., Anton L., Aloy M. A., Muller W., Mart M., Miralles J. A., \& Ibanez J. M., 2005, $A \mathscr{E} A S, 436,503$

Massaglia, S., Bodo, G., Mignone, A., \& Rossi, P. 2008, Jets from Young Stars III - Numerical $M H D$ and Instabilities, Lecture Notes in Physics 754, Springer

Mignone, A., Bodo, G., Massaglia, S., Matsakos, T., Tesileanu, O., Zanni, C., \& Ferrari, A. 2007, ApJS, 170, 228

Mignone, A. \& McKinney, J. C. 2007, MNRAS, 378, 1118

Mignone, A., Rossi, P., Bodo, G., Ferrari, A., \& Massaglia, S. 2010, MNRAS, 402, 7

Rossi, P., Mignone, A., Bodo, G., Massaglia, S., \& Ferrari, A. 2008, A\&AS, 488, 795

Rossi, P., Mignone, A., Bodo, G., Massaglia, S., \& Ferrari, A. 2010, in preparation 